\title{
Physical Exam Domain
}

National Cancer Institute

\section{Source}

National Cancer Institute. Physical Exam Domain. NCI Thesaurus. Code C49608.

A subject domain utilized for the submission of information encompassing and representing data, vocabulary or records related to physical exam. 